\title{
Mechanisms of Homocysteine-Induced Glomerular Injury and Sclerosis
}

\author{
Fan Yi Pin-Lan Li \\ Department of Pharmacology and Toxicology, Medical College of Virginia, Virginia Commonwealth University, \\ Richmond, Va., USA
}

\section{Key Words}

Hyperhomocysteinemia $\cdot$ Glomerulus $\cdot$ Sulfur amino acids · Oxidative stress $\cdot$ Mesangial cells $\cdot$ Podocytes $\cdot$ Proteinuria

\section{Abstract}

Hyperhomocysteinemia (hHcys) has been recognized as a critical risk or pathogenic factor in the progression of endstage renal disease (ESRD) and in the development of cardiovascular complications related to ESRD. Recently, evidence is accumulating that hHcys may directly act on glomerular cells to induce glomerular dysfunction and consequent glomerular sclerosis, leading to ESRD. In this review, we summarize recent findings that reveal the contribution of homocysteine as a pathogenic factor to the development of glomerular sclerosis or ESRD. In addition, we discuss several important mechanisms mediating the pathogenic action of homocysteine in the glomeruli or in the kidney, such as local oxidative stress, endoplasmic reticulum stress, homocysteinylation, and hypomethylation. Understanding these mechanisms may help design new approaches to develop therapeutic strategies for treatment of hHcys-associated end-organ damage and for prevention of deterioration of kidney function and ultimate ESRD in patients with hypertension and diabetes mellitus or even in aged people with hHcys.
\end{abstract}

\section{Homocysteine (Hcys) Metabolism and Hyperhomocysteinemia (hHcys)}

Hcys as a thiol-containing amino acid has gained great notoriety, since elevation of its plasma concentrations, a condition known as hHcys, is correlated with many different diseases, in particular cardiovascular diseases [1-3], stroke [4], neurodegenerative diseases [5], neural tube defects $[6,7]$, and end-stage renal disease (ESRD) [8-12]. As shown in figure 1, Hcys is mainly generated from methionine which contains a methyl group that is activated through the transformation to S-adenosylmethionine (SAM). SAM is a major methyl group donor for various methylation reactions. When the methyl group is transferred by methyltransferases to respective acceptors, SAM is converted to S-adenosylhomocysteine (SAH) and then subsequently hydrolyzed by SAH hydrolase to form Hcys and adenosine.

It is well known that Hcys is metabolized by two alternative pathways, including its remethylation and transsulfuration. The remethylation pathway regenerates methionine by methylenetetrahydrofolate reductase (MTHFR) using Hcys as substrate and folate and vitamin $\mathrm{B}_{12}$ as cofactors. This remethylation cycle may occur in all mammalian cells or tissues which could control the concentrations of Hcys within these cells or tissues. Another pathway responsible for the Hcys metabolism is its transsulfuration which is catalyzed by two sequentially acting enzymes, namely cystathione- $\beta$-synthase (CBS) and $\gamma$-cystathionase. In this pathway, Hcys first undergoes condensation with serine to form cystathionine by

\begin{tabular}{ll}
\hline KARGER & $\odot$ 2007 S. Karger AG, Basel \\
$\begin{array}{l}\text { Fax }+41613061234 \\
\begin{array}{l}\text { E-Mail karger@karger.ch } \\
\text { www.karger.com }\end{array}\end{array}$ & $\begin{array}{l}\text { Accessible online at: } \\
\text { www.karger.com/ajn }\end{array}$
\end{tabular}

Pin-Lan Li, MD, PhD

Department of Pharmacology and Toxicology, Medical College of Virginia Virginia Commonwealth University, 410 North 12th Street, PO Box 980613 Richmond, VA 23298 (USA)

Tel. +1 804828 4793, Fax +1 804828 4794, E-Mail pli@maill.vcu.edu 
Fig. 1. Homocysteine metabolism. Methionine is activated by ATP to S-adenosylmethionine (SAM) which serves as a universal donor for methyl transfer reactions. $\mathrm{S}$-adenosylhomocysteine (SAH) is produced as a product of methyl transfer reactions that utilize SAM as a methyl donor. Homocysteine is formed from the reversible hydrolysis of SAH. The levels of homocysteine are regulated by remethylation of homocysteine to methionine by the enzyme methionine synthase (MS) and transsulfuration of homocysteine to cystathionine by the enzyme cystathionine $\beta$ synthase (CBS).

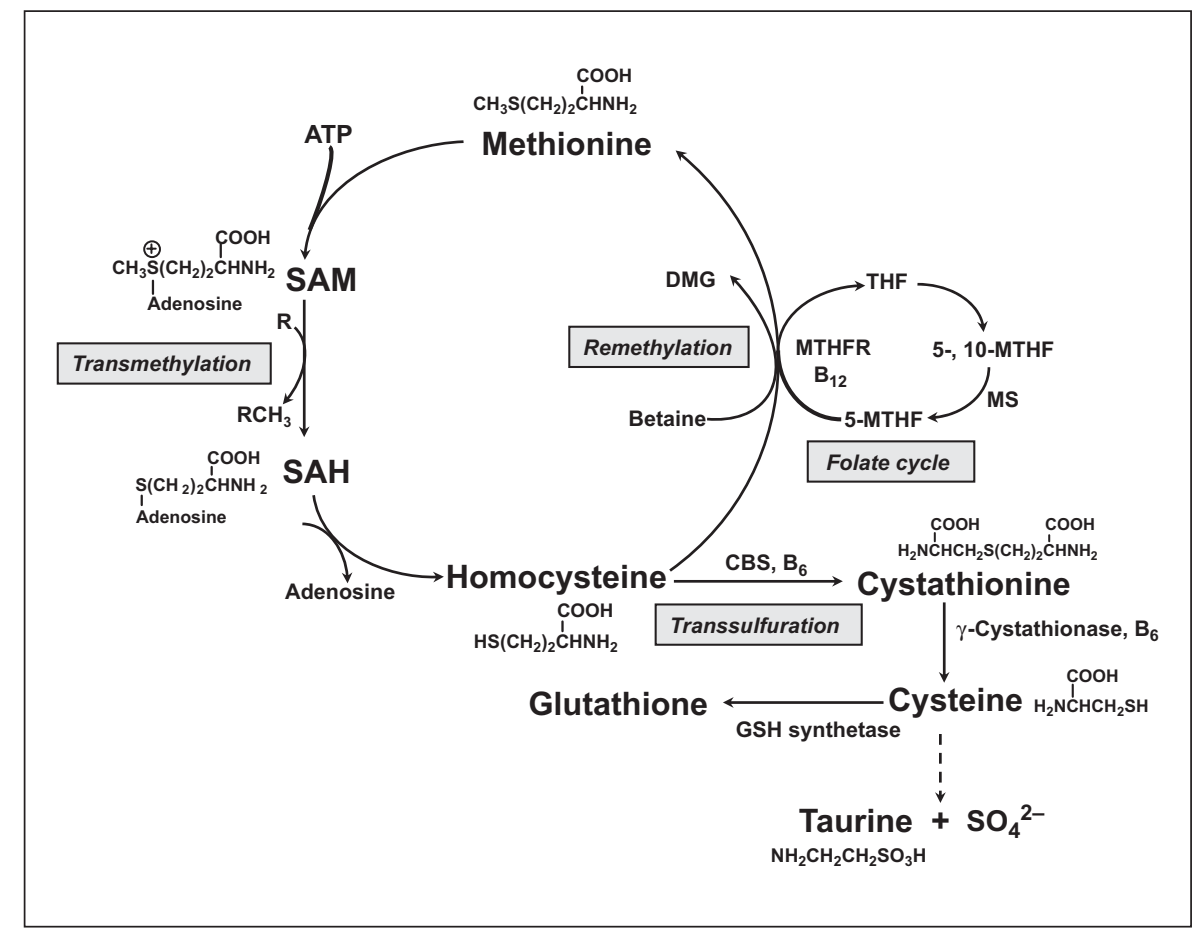

CBS. Then, cystathionine is broken down into cysteine by $\gamma$-cystathionase. Finally, cysteine is metabolized into taurine and sulfate or transferred into glutathione. The synthesis of cystathionine is an irreversible enzymatic reaction which occurs primarily in liver, kidney, small intestine, and pancreas [13]. Although two enzymes are involved in this metabolic pathway of Hcys, CBS is rate limiting in this transsulfuration metabolism of Hcys. In the kidney, Hcys is primarily transsulfurated, and deficiency of this renal transulfuration importantly contributes to the elevation of plasma Hcys under different physiological or pathological conditions, such as hypertension, diabetes mellitus, aging, or ESRD [14, 15].

There are reduced and oxidized forms of Hcys in human and animal plasma [16]. More than $90 \%$ of the total plasma Hcys is oxidized Hcys that is bound to proteins, such as albumin and hemoglobin. The rest of the oxidized Hcys exists as a disulfide form, including homocystine (Hcys-S-S-Hcys) and cysteinylhomocysteine (Cys-S-SHcys). Another small fraction of oxidized Hcys is Hcys thiolactone, a Hcys metabolite which only represents about $0.4 \%$ of the total plasma Hcys, but is very active in regard to the detrimental actions of Hcys. Only about $2 \%$ of the total Hcys in plasma exists as a free or reduced form. In general, hHcys is defined as an increase in the plasma level of total Hcys which is the sum of all Hcys forms that

Hyperhomocysteinemia and End-Stage Renal Disease exist in plasma. Structures of some major species of Hcys in the plasma are summarized in figure 2. In humans, the total plasma Hcys concentration is normally $<10 \mu \mathrm{M}$. Clinically, a mild hHcys is defined as a plasma total Hcys level $>10 \mu \mathrm{M}$, but $<16 \mu \mathrm{M}$. A plasma total Hcys level in the range of $16-30 \mu \mathrm{M}$ is considered moderate hHcys and one in the range of 30-100 $\mu \mathrm{M}$ intermediate hHcys. If the plasma total Hcys level is $>100 \mu \mathrm{M}$, a severe hHcys may be diagnosed [12, 17]. Many diseases or metabolic deficiency may cause hHcys, such as mutations of the gene encoding key enzymes of the Hcys metabolism such as CBS and methylenetetrahydrofolate reductase, decreased intake or intestinal absorption of vitamin $B$ or other cofactors like folic acid and vitamin $\mathrm{B}_{12}$, drug administration like L-DOPA, or chronic renal failure $[14,18]$.

\section{hHcys and ESRD}

hHcys was originally reported as a risk factor for atherosclerosis [19-21] based on observational data that link increased plasma Hcys levels to premature arteriosclerotic diseases [22]. Later, numerous epidemiological and clinical case-control studies have observed a significant positive association between plasma Hcys levels and cardiovascular diseases, including hypertension, stroke [23, 


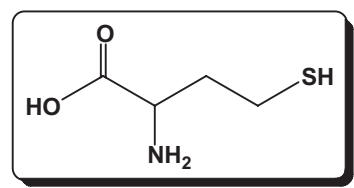

Homocysteine

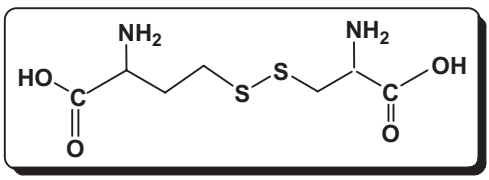

Cysteine-homocysteine

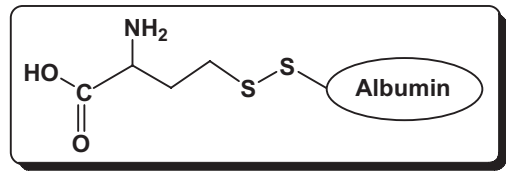

S-homocysteine-albumin

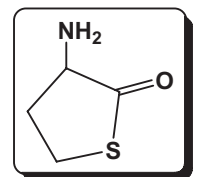

Homocysteine thiolactone

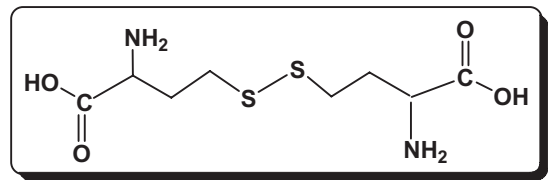

Homocysteine-homocysteine

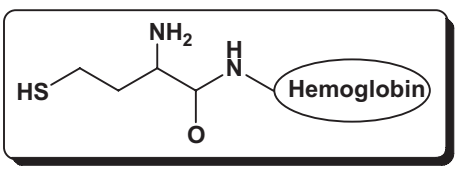

N-homocysteine-hemoglobin

Fig. 2. Major species of homocysteine present in human and animal plasma.

24], and coronary arterial disease [25]. In this regard, Boushey et al. [26] have reported that the risk associated with a $5-\mu \mathrm{M}$ increase in plasma total Hcys is similar to that associated with a $0.5-\mathrm{mm}$ increase in the plasma cholesterol concentration. A meta-analysis of prospective studies has also shown that a $25 \%$ lower plasma total Hcys concentration is associated with an $11 \%$ lower risk of ischemic heart disease and a 19\% lower risk of stroke [27]. In addition, a strong positive relationship between Hcys levels and mortality in patients with established cardiovascular diseases has also been observed by Nygard et al. [28]. All these data have indicated that a deranged Hcys metabolism may increase the incidence or prevalence of cardiovascular diseases in general populations.

As reviewed by Brattström and Wilcken [29], almost every study in which the plasma Hcys level was measured in patients with renal diseases has shown a highly significant positive correlation between serum creatinine and Hcys concentrations. The plasma total Hcys levels increase in patients with moderate renal failure and can be $>100 \mu \mathrm{M}$ in patients with ESRD [11]. The prevalence of hHcys in patients with dialysis therapy is $>85 \%$, and in these patients the plasma Hcys levels may remain three to five times higher than the normal level. It has been proposed that hHcys is a crucial element in the pathogenesis of uremic cardiovascular complications [30-32].
Increased plasma Hcys levels during chronic renal failure or ESRD might be caused by an increased production via transmethylation and decreased metabolism or removal. The latter includes decreases in its transsulfuration, remethylation, and renal excretion. However, it is still debated as to whether an increased plasma Hcys level in patients with ESRD is due to a reduction of the renal metabolic clearance or an extrarenal metabolic disturbance [33]. It also remains unknown what renal mechanisms could be responsible for elevated plasma Hcys concentrations during ESRD or other kidney diseases. In this regard, early studies have indicated that increased plasma Hcys levels are attributed to a reduction of the glomerular filtration rate (GFR) [31, 34]. However, recent studies have challenged this view, since Hcys may be filtrated in very small amounts due to its binding to plasma proteins. Many studies indeed demonstrated that clinically stable renal transplant recipients have an excess prevalence of hHcys, suggesting that improvement of the GFR in these patients does not completely restore plasma Hcys to normal $[9,29]$. Similarly, in ESRD patients receiving hemodialysis therapy, improvement of the renal function could not restore plasma Hcys concentrations to normal levels $[35,36]$. In some patients with hypertension, moreover, long-term diuretic therapy results in hHcys, but their GFR or renal functions are normal [3]. More directly, a 
study performed in hypertensive patients $[12,37] \mathrm{ob}$ served that the fractional extraction of Hcys across the kidney is related positively to renal plasma flow but not to GFR. All these data have strongly indicated that elevated Hcys levels in patients with ESRD or hypertension are at least not solely dependent upon a decrease in GFR. An intrinsic metabolic derangement may also be importantly involved in elevation of plasma Hcys levels which may be related to decreased renal transsulfuration. Our recent studies done in a hypertensive rat model, Dahl salt-sensitive rats, have demonstrated that abnormal renal transsulfuration by renal tubules is a major cause of hHcys in these rats [14], indicating that the defect in renal transsulfuration may be responsible for the development of hHcys in salt-sensitive hypertensive individuals. In addition, extrarenal metabolic disturbances of Hcys have also been reported to contribute to the occurrence of hHcys in ESRD. These metabolic disturbances are associated with a generalized downregulation of the methionine cycle and its catabolism which may be the inhibitory action of various metabolites accumulated or retained during ESRD, such as SAH, sulfate, and dimethylglycine.

Although hHcys is considered a critical pathogenic factor in the progression of ESRD and in the development of cardiovascular complications related to ESRD, some recent studies have shown that plasma Hcys levels may also be inversely related to the clinical outcome of ESRD which is now called 'reverse epidemiology' of hHcys in ESRD [38-40]. Along with the line, low plasma Hcys levels may be associated with a worse prognosis or mortality in ESRD. However, this reverse association of plasma Hcys levels with the clinical outcome in ESRD patients is not only observed for hHcys, but also for many other cardiovascular risk factors, including blood pressure, cholesterol, and body size. Several possible explanations have been given for this reverse epidemiology in ESRD which include survival bias and discrepancies between competitive risk factors. Among the most important confounding factors to influence Hcys levels in ESRD patients are hypoalbuminemia, malnutrition, inflammation, and diabetes mellitus [41]. In particular, malnutrition is considered a critical factor resulting in this reverse phenomenon, since it leads to cachexia, now called 'malnutritioninflammation-cachexia syndrome' $[42,43]$. Under this condition, a reverse association of plasma Hcys levels does not mean that high plasma Hcys levels produce beneficial action, but rather indicate that high Hcys levels may reflect a better cachexia status of patients with ESRD.

Hyperhomocysteinemia and End-Stage Renal Disease

\section{Potential Mechanisms of Hcys-Induced Toxicity in Glomeruli}

Despite substantial evidence indicating the association between hHcys and ESRD, the pathogenic role of increased plasma Hcys levels in the progression of ESRD remains controversial. Given the similarity of pathological changes between glomerular injury and Hcys-induced arterial damages, such as endothelial injury, cell proliferation or growth, increased matrix formation, and aggregated proteoglycan $[20,22]$, it is assumed that an increase in plasma Hcys levels may also directly act on glomerular cells, resulting in glomerular injury and ultimate sclerosis. Moreover, an impaired renal function will lead to a further increase in plasma Hcys levels which in turn exaggerates the progression of glomerular injury, resulting in a vicious cycle and consequent glomerulosclerosis and ESRD. In addition, Hcys may also produce detrimental actions or increase the risk of cardiovascular disease by decreasing plasma or tissue adenosine levels $[44,45]$. It is well known that adenosine evokes several biological actions in the cardiovascular system and participates in the regulation of the renal function, including renal glomerular perfusion [46]. We have demonstrated [45] that chronic elevations of the plasma Hcys concentrations in rats resulted in arteriosclerotic changes and glomerular dysfunction and sclerosis accompanied by a sustained low level of plasma adenosine. It is possible that the sclerotic effect of hHcys is associated with decreased adenosine. Indeed, it was demonstrated [47] that decreased adenosine levels are associated with enhanced proliferation or growth of vascular smooth muscle cells and sclerotic changes in arteries or glomeruli. Decreased adenosine concentrations during hHcys may be due to an enhanced activity of a bidirectonal enzyme, SAH hydrolase. As discussed above, Hcys is formed by hydrolysis of SAH via SAH hydrolase. At the same time, a molecule of adenosine is produced during this enzyme reaction. However, under certain conditions, this enzyme could catalyze the condensation of Hcys and adenosine to form $\mathrm{SAH}$. In particular, when adenosine, Hcys, or both increased, the SAH synthesis was markedly enhanced [48], resulting in a reduction of adenosine levels in blood or in different tissues. In a recent review, Deussen et al. [44] have summarized recent findings regarding the pathogenicity of adenosine in hHcys.

With respect to the direct action of Hcys to damage cells or induce sclerotic changes, recent studies focus on several important cellular and molecular mechanisms. Below we summarize some of these recent findings re-

Am J Nephrol 2008;28:254-264 
lated to its damaging actions on glomerular cells and the possible role in the development of glomerular sclerosis or chronic renal failure. It is our belief that understanding these mechanisms will help further clarify the pathogenesis of ESRD under different pathological processes, such as hypertension and diabetes mellitus. Most importantly, some new directions for studies on Hcys-induced glomerular injury and prevention or treatment of hHcys-associated ESRD are suggested during discussion of these mechanisms.

\section{Local Oxidative Stress}

In numerous studies, oxidative stress in local tissues or cells has been implicated in the pathological actions of hHcys. Hcys may reduce nitric oxide production and directly trap nitric oxide, thereby increasing tissue superoxide $\left(\mathrm{O}_{2}{ }^{-}\right)$levels. It can also enhance the production of reactive oxygen species (ROS) by auto-oxidation of itself and reduction of antioxidant enzyme activities, such as superoxide dismutase, thioredoxin/thioredoxin reductase, and glutathione peroxidase-1 [12, 16, 29, 49, 50]. In addition, redox regulation is also considered an important mechanism in the regulation of the Hcys metabolism [49]. With respect to the redox regulation of Hcys-metabolizing enzymes, it seems that several enzymes involved in the Hcys metabolism are redox sensitive, such as methionine synthase in remethylation and CBS, $\gamma$-glutamylcysteine ligase, and glutathione synthetase in transsulfuration [49, 51-53]. For detail information regarding this redox regulation of the Hcys metabolism, the readers are directed to the recent review by Zou and Banerjee [49]. Here we will mainly focus on the role of the redox mechanism in Hcys-induced pathogenic actions in hHcys.

\section{NADPH Oxidase}

Although multiple enzymes may contribute to oxidative stress in different tissues or cells, it is generally accepted that NADPH oxidase is a major source of ROS in many nonphagocytic cells [54-57]. In the kidney, $\mathrm{O}_{2}{ }^{-}$ produced via NADPH oxidase can be detected in fibroblasts, endothelial cells, vascular smooth muscle cells, mesangial cells, tubular cells, and podocytes [56-58]. This $\mathrm{O}_{2}^{--}$-producing enzyme in the kidney has similar characteristics to an NADPH oxidase identified in phagocytes. It consists of a membrane-bound flavocytochrome $b_{558}$, the three cytosolic subunits $\mathrm{p} 47^{\text {phox }}, \mathrm{p} 67^{\text {phox }}$, and $\mathrm{p} 40^{\text {phox }}$, and a small GTPase, Rac1 or Rac2 $[59,60]$. The membrane-bound flavocytochrome $b_{558}$ is a complex of two membrane subunits, namely a flavin- and hemebinding glycoprotein gp91phox and a smaller subunit p $22^{\text {phox }}$. In the past few years, a family of gp $91^{\text {phox }}$-like proteins, termed the nonphagocytic $\mathrm{NAD}(\mathrm{P}) \mathrm{H}$ oxidase (NOX) proteins, has been identified, including NOX1, NOX2 (gp91 $\left.{ }^{\text {phox }}\right)$, NOX3, NOX4, and NOX5. In addition, DUOX1 and DUOX2 are 175 - to $180-\mathrm{kDa}$ proteins that have a domain homologous to gp91 ${ }^{\text {phox }}$ as well as an additional peroxidase domain. All isoforms of NOX are expressed in the kidney, and NOX4 is originally described as a renal oxidase (renox) [55, 59]. To produce $\mathrm{O}_{2}{ }^{-}$, NADPH oxidase is normally activated by $4^{p^{h o x}}$ phosphorylation and translocation, Rac GTPase activation, or protein-protein interactions [61].

Recent studies have demonstrated that NADPH oxidase is importantly involved in the progressive glomerular injury associated with hHcys [62-64]. In cultured rat mesangial cells, L-Hcys (40-160 $\mu \mathrm{M}$, active form of Hcys) markedly increased the mRNA levels of tissue inhibitor of metalloproteinase- 1 and led to accumulation of collagen type I which was accompanied by enhanced cell proliferation and NADPH oxidase activity. These Hcys-induced biochemical and functional changes could be substantially blocked by an NADPH oxidase inhibitor, DPI, or a superoxide dismutase mimetic, hydroxyl-tetramethylpiperidine-oxyl. Moreover, blockade of the NADPH oxidase subunit $\mathrm{p} 22^{\text {phox }}$ by its antisense oligodeoxynucleotide also eliminated the increase in NADPH oxidase activity induced by L-Hcys [65]. In the rat model of hHcys induced by a folate-free diet, glomerular sclerosis occurred which is characterized by enhanced local oxidative stress, mesangial expansion, podocyte dysfunction, and fibrosis. When these rats with $\mathrm{hHcys}$ were treated with the NADPH oxidase inhibitor apocynin, the glomerular injuries were significantly attenuated $[63,66]$, as shown by decreased urinary albumin excretion and reduced glomerular damage index. It is concluded that Hcys-induced local oxidative stress and consequent cell dysfunction and extracellular matrix (ECM) metabolism in glomerular cells are associated with enhanced NADPH oxidase activity. We also demonstrated that Hcys-induced activation of $\mathrm{NADPH}$ oxidase is associated with increased de novo ceramide synthesis and consequent enhancement of Rac GTPase activity [67]. Other studies have also confirmed that Hcys-induced ROS is associated with NADPH oxidase. In addition, Hcys has been reported to cause mesangial apoptosis via ROS generation and p38-mitogen-activated protein kinase activation [62]. Taken together, these findings support the view that local oxidative stress or a change in redox status in glomeruli due to activation of 
NADPH oxidase importantly contributes to glomerular injury and ultimate glomerular sclerosis in hHcys.

The role of NADPH-oxidase-derived $\mathrm{O}_{2}{ }^{--}$in mediating Hcys-induced cell injury or sclerosis is also confirmed in studies using other tissues or cells [68]. Dai et al. [69] have shown that Hcys-induced upregulation and translocation of Ref-1 is due to ROS production via NADPH oxidase. Increased Ref-1 enhances nuclear factor-kappa B activity and monocyte chemoattractant protein-1 secretion in monocytes/macrophages, accelerating the development of atherosclerosis [69]. There is also evidence that folic acid inhibits the Hcys-induced $\mathrm{O}_{2}{ }^{\cdot-}$ production via NADPH oxidase and nuclear factor-kappa $B$ activation in macrophages which may constitute a solid basis for folic acid treatment in Hcys-induced oxidative stress and inflammatory responses [70]. All these results obtained from both renal and nonrenal studies suggest that $\mathrm{NADPH}$ oxidase is an important intermediate enzyme, mediating Hcys-induced local oxidative stress and consequent cell injury and tissue sclerosis, such as atherosclerosis and glomerular sclerosis.

\section{Mitochondria-Derived ROS}

Another important source of $\mathrm{O}_{2}{ }^{\cdot-}$ is the electron transport chain in mitochondria [71], where complexes I and III are primarily responsible for the generation of $\mathrm{O}_{2}{ }^{-}$ [72]. Under certain conditions, such as hypoxia, mitochondria produce $\mathrm{O}_{2}{ }^{--}$from the semiquinone form of coenzyme $\mathrm{Q}$ and a reduced component of NADH dehydrogenase [73]. Mitochondrial $\mathrm{O}_{2}{ }^{--}$may be converted into $\mathrm{H}_{2} \mathrm{O}_{2}$ by manganese superoxide dismutase, and the latter may freely diffuse into cytosol or other cellular compartments and thereby produce various injurious effects. Evidence is increasing that a continuous release of mitochondria-derived $\mathrm{H}_{2} \mathrm{O}_{2}$ or other ROS is an important mechanism mediating the pathogenic action of Hcys in cardiovascular diseases [74, 75]. For example, Tyagi et al. [68] have shown a positive relationship between cellular mitochondrial oxidative stress and systemic hypertension in hHcys. Sethi et al. [76] also reported that a combined metabolic burden of Hcys and high glucose stimulates ET1 synthesis in endothelial cells via a mitochondrial ROSdependent mechanism and thereby results in cell dysfunction. Given the similarity of pathological changes at the cellular or molecular levels in glomerular injury and arterial damages, mitochondria-derived ROS may also be importantly involved in the initiation and progression of Hcys-induced glomerular sclerosis. Further studies are needed to provide direct evidence demonstrating this mitochondrial mechanism in glomerular cells.

Hyperhomocysteinemia and End-Stage Renal Disease

\section{Endoplasmic Reticulum (ER) Stress}

Recently, the Hcys-induced ER stress response has received much attention, and increasing evidence indicates that this ER stress may represent an important fundamental mechanism mediating the pathogenic action of hHcys [77-79]. The ER is an intracellular compartment that plays a critical role for protein synthesis and folding, calcium storage, and calcium release. It also serves as a site of biosynthesis for steroids, cholesterol, and other lipids. Proteins that are translocated into the ER lumen undergo posttranslational modifications and folding required for optimal function. Properly folded proteins are allowed to reach their destiny via the secretory pathway, whereas unfolded and misfolded proteins are exported or dislocated from the ER and then degraded by cytoplasmic proteasomes [80, 81]. ER stress is referred to as a condition under which unfolded and misfolded proteins are accumulated. Accumulation of these unfolded or misfolded proteins activates an unfolded protein response (UPR) which includes increased expressions of UPR-responsive genes, reduced global protein translation, and unfolded protein degradation. Recent studies have demonstrated that Hcys causes ER stress by disruption of disulfide bond formation and misfolding proteins. In addition, elevation of intracellular Hcys levels increases the expression of several ER stress response genes, including GRP78, GRP94, Herp, GADD153, and RTP [77-79, 82], among which increased GADD153 expression may activate cell death [83]. Other mechanisms by which Hcys induces cell injury through ER stress may be associated with activation of IRE-1, a signaling molecule that leads to a rapid and sustained activation of JNK protein kinases and cell apoptosis $[84,85]$ or dysregulation of the sterol response to Hcys. This multiple disturbance of protein and lipid metabolisms during Hcys-induced ER stress has been indeed observed as a mechanism for tissue or organ sclerotic changes in CBS-deficient patients with severe hHcys and in a diet-induced mouse model of hHcys [77].

Specific to the kidney, the pathophysiological role of ER stress in glomerular injury or chronic renal failure both in vitro and in vivo has also been reported. Inagi et al. provided evidence that complement attack against podocytes induces ER injury which is associated with upregulation of ER stress proteins such as GRP78 and GRP94 both in vivo and in vitro. By analysis of the expression of ER stress, the authors demonstrated that inducible proteins such as GRP78, GRP94, and ORP150 in cultured podocytes treated with tunicamycin, A23187, 
SNAP, and hyperglycemia are upregulated. Using a transgenic rat model that overexpresses a serine protease inhibitor, megsin gene [86, 87], ER stress is detected in isolated glomeruli as shown by flooding of the proteinhandling system and subsequent accumulation of polymerizing megsin within the ER of podocytes. This ER stress of podocytes is accompanied by proteinuria and deterioration of the renal function [86, 87]. It is clear that accumulation of proteins in the podocyte ER due to its stress contributes to the development of podocyte injury and proteinuria [88]. Given that Hcys is a strong stimulating factor of ER stress and podocyte injury [66], it is possible that ER stress is an important pathogenic mechanisms in Hcys-induced sclerosis.

\section{Homocysteinylation}

Protein homocysteinylation is recently emerging as an important cellular mechanism responsible for Hcys-induced toxicity in a variety of cells or tissues. It has been reported that there are many plasma proteins that may be homocysteinylated in vitro and in vivo [89]. Protein homocysteinylation occurs through several different mechanisms, among which posttranslational acylation of free amino groups (protein-N-homocysteinylation) is one of the most important processes. This protein-N-linked Hcys is formed when Hcys thiolactone enters the blood circulation and reacts with an epsilon amino group of lysine via its activated carbonyl group to form a stable amide bond. Another type of protein homocysteinylation occurs through the formation of a covalent $-S-S-$ bond by its reaction with cysteine residues (protein-S-homocysteinylation) [90-92].

Protein homocysteinylation may lead to its molecular damage and function loss. Among a number of homocysteinylated proteins, several are importantly involved in the sclerotic process in arteries, brain, or glomeruli. For example, low-density lipoprotein-N-homocysteinylation may cause protein aggregation, accumulation of intracellular cholesterol, and formation of 'foam cells' [93]. This homocysteinylated low-density lipoprotein also induces oxidative stress in cultured human aortic endothelial cells which may lead to endothelial dysfunction, a key event of artherosclerotic plaque fromation [94, 95]. Another homocysteinylated protein is fibronectin, an ECM protein. This fibronectin-S-homocysteinylation impairs fibronectin-fibrin interaction, resulting in dysfunction of cell adhesion and migration and tissue remodeling [96]. Importantly, this homocysteinylated fibronectin may lead to abnormal ECM metabolism, promoting the development of sclerotic processes.

There is considerable evidence that protein homocysteinylation participates in glomerular sclerosis and in the development of ESRD. In this regard, Perna et al. [90] have reported that plasma homocysteinylation increased in ESRD patients subject to hemodialysis, where both protein-N-homocysteinylation and protein-S-homocysteinylation are significantly higher as compared with normal subjects. It was demonstrated [97] that in patients with terminal renal failure without dialysis S-homocysteinylation and N-homocysteinylation reactions of proteins are significantly enhanced which may contribute to the atherogenesis and progression of ESRD in these patients. Another interesting mechanism that possibly mediates glomerular injury or sclerosis induced by homocysteinylation is the irreversible homocysteinylation of long-lived proteins in connective tissues. In this regard, an intensively studied extracellular connective tissue protein, fibrillin-1, and epidermal growth factor-like domain present in many other extracellular proteins are especially susceptible to Hcys (and presumably Hcy thiolactone) attacks [98]. This homocysteinylation of these proteins may cause abnormal metabolism of the ECM, promoting the progression of glomerular sclerosis [99, 100].

\section{Hypomethylation}

Hcys-induced hypomethylation has been recognized as a pathogenic mechanism of Hcys even at an early stage, when this amino acid was considered a risk factor for cardiovascular disease. There is increasing evidence that this hypomethylation during hHcys also participates in the progression of ESRD $[32,93]$. SAH as a potent competitive inhibitor of methyltransferases may result in hypomethylation which causes DNA damage and cell apoptosis and induces tissue inflammation and sclerotic responses [101]. In patients with ESRD, a hypomethylation status of cellular proteins and DNA can be detected, as shown by high SAH intracellular concentrations, low SAM/SAH concentration ratios $[18,102]$, and inhibited protein methylation and repair [103]. This supports the view that the toxic actions of Hcys may be mediated by DNA hypomethylation in ESRD. It is clear that this hypomethylation induced by hHcys may alter the function of various cellular proteins, DNA, and gene expression and thereby initiate or promote the sclerotic process [12, 15]. For more detailed information about the role of Hcys- 
Fig. 3. Summarized mechanisms of homo-

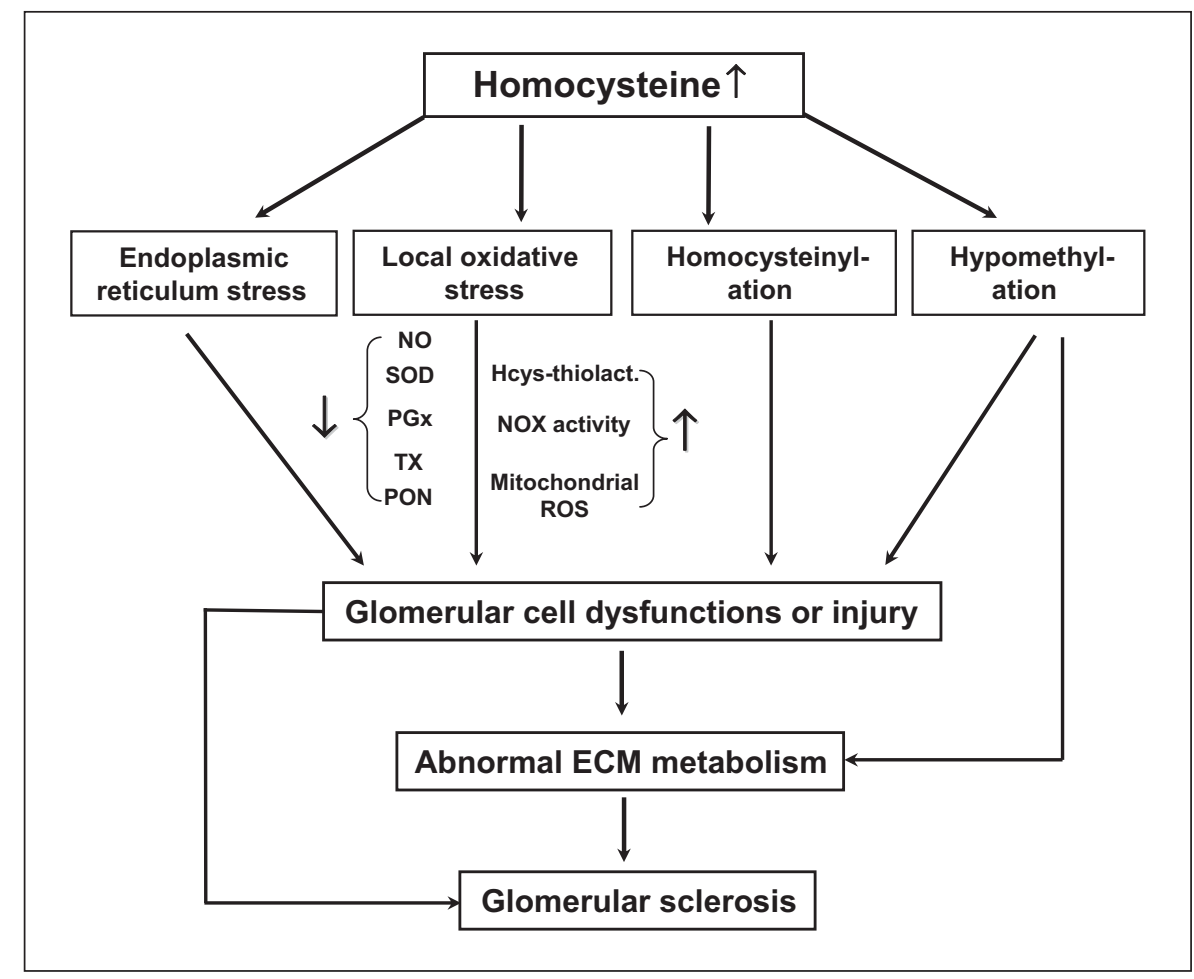
cysteine-induced toxicity in the kidney.

induced hypomethylation in the progression of ESRD, the recent review by van Guldener et al. [15] is suggested for further reading.

\section{Conclusions and Perspective}

This review provides evidence that supports the role of Hcys in the development of glomerular sclerosis or ESRD and outlines several important cellular and molecular mechanisms by which Hcys may produce pathogenic action on glomeruli to induce glomerular injury or sclerosis. These mechanisms include the role of local oxidative stress, ER stress, homocysteinylation, and hypomethylation. Due to space limitations, some other interesting topics related to the possible mechanisms mediating Hcys-induced glomerular toxicity are not included, such as activation of proinflammatory factors, increased homocysteine thiolactone levels, and Hcys-induced abnormal mitochondrial biogenesis. Figure 3 summarizes all discussed mechanisms to present an integrate picture for understanding pathogenesis of Hcys-induced glomerular injury and progression of ESRD. In perspective, further studies are much needed to provide more convincing evidence demonstrating the contribution of these mechanisms to glomerular injury or sclerosis in in vivo animal experiments and clinical studies. Some key targets in these pathogenic pathways for Hcys-induced injury or sclerosis must be identified to direct toward prevention or treatment of ESRD associated with hHcys which is particularly important since so far there are no efficient Hcys-lowering and Hcys-detoxifying strategies being used in chronic kidney disease patients. Although folic acid and some B vitamins may enhance Hcys metabolism or decrease Hcys efflux into the plasma compartment, many studies have demonstrated that such folate- or B-vitamin-based therapy is insufficient to override the primary defect of the Hcys metabolism in ESRD patients to restore plasma Hcys to a normal level. For example, a very recent clinical study [104] reported that three large, randomized, controlled trials of patients with preexisting cardiovascular disease and two smaller, randomized, controlled trials of patients with kidney failure failed to demonstrate any cardiovascular benefit from Hcys-lowering vitamins. Therefore, understanding the mechanisms responsible for Hcys-induced specific pathological actions may provide clues to develop new therapeutic strategies beyond lowering the plasma Hcys levels. It should be noted that despite some controversial clinical results or reports about the beneficial effect of Hcys-low- 
ering therapy, conclusions should be drawn with caution, unless the plasma Hcys levels in patients with chronic renal failure or ESRD could become normal. We hope that this review could provide some useful information to help find valuable ideas in advancing this area, in particular, in developing new therapeutic strategies for the prevention of hHcys-associated glomerular injury or sclerosis.

\section{Acknowledgments}

This study was supported by grants DK54927, HL070726, and HL57244 from the National Institutes of Health.

\section{References}

1 Anderson JL, Muhlestein JB, Horne BD, Carlquist JF, Bair TL, Madsen TE, Pearson RR: Plasma homocysteine predicts mortality independently of traditional risk factors and C-reactive protein in patients with angiographically defined coronary artery disease. Circulation 2000;102:1227-1232.

$\checkmark 2$ Cavalca V, Cighetti G, Bamonti F, Loaldi A, Bortone L, Novembrino C, De Franceschi M, Belardinelli R, Guazzi MD: Oxidative stress and homocysteine in coronary artery disease. Clin Chem 2001;47:887-892.

$\checkmark 3$ Perry IJ: Homocysteine, hypertension and stroke. J Hum Hypertens 1999; 13:289-293.

$\checkmark 4$ Yoo JH, Lee SC: Elevated levels of plasma homocyst(e)ine and asymmetric dimethylarginine in elderly patients with stroke. Atherosclerosis 2001;158:425-430.

5 Nahlawi M, Seshadri N, Boparai N, Naso A, Jacobsen DW, McCarthy P, Young J, Robinson $\mathrm{K}$ : Usefulness of plasma vitamin $\mathrm{B}_{6}, \mathrm{~B}_{12}$, folate, homocysteine, and creatinine in predicting outcomes in heart transplant recipients. Am J Cardiol 2002;89:834-837.

6 Mills JL, Scott JM, Kirke PN, McPartlin JM, Conley MR, Weir DG, Molloy AM, Lee YJ: Homocysteine and neural tube defects. J Nutr 1996;126:756S-760S.

7 Sachdev PS, Valenzuela M, Wang XL, Looi JC, Brodaty H: Relationship between plasma homocysteine levels and brain atrophy in healthy elderly individuals. Neurology 2002; 58:1539-1541.

-8 Dennis VW, Robinson K: Homocysteinemia and vascular disease in end-stage renal disease. Kidney Int Suppl 1996;57:S11-S17.

$\checkmark 9$ Ducloux D, Motte G, Challier B, Gibey R, Chalopin JM: Serum total homocysteine and cardiovascular disease occurrence in chronic, stable renal transplant recipients: a prospective study. J Am Soc Nephrol 2000;11: 134-137.

10 Gupta A, Robinson K: Hyperhomocysteinaemia and end-stage renal disease. J Nephrol 1997;10:77-84.

-11 Guttormsen AB, Ueland PM, Svarstad E, Refsum H: Kinetic basis of hyperhomocysteinemia in patients with chronic renal failure. Kidney Int 1997;52:495-502.
12 Perna AF, Ingrosso D, Satta E, Lombardi C, Acanfora F, De Santo NG: Homocysteine metabolism in renal failure. Curr Opin Clin Nutr Metab Care 2004;7:53-57.

-13 Brosnan JT, Jacobs RL, Stead LM, Brosnan ME: Methylation demand: a key determinant of homocysteine metabolism. Acta Biochim Pol 2004;51:405-413.

14 Li N, Chen L, Muh RW, Li PL: Hyperhomocysteinemia associated with decreased renal transsulfuration activity in Dahl S rats. Hypertension 2006;47:1094-1100.

15 van Guldener C, Stam F, Stehouwer CD: Hyperhomocysteinaemia in chronic kidney disease: focus on transmethylation. Clin Chem Lab Med 2005;43:1026-1031.

16 Jacobsen DW: Homocysteine and vitamins in cardiovascular disease. Clin Chem 1998; 44:1833-1843.

17 Kang SS, Wong PW, Malinow MR: Hyperhomocyst(e)inemia as a risk factor for occlusive vascular disease. Annu Rev Nutr 1992; 12:279-298.

- 18 Perna AF, Capasso R, Lombardi C, Acanfora F, Satta E, Ingrosso D: Hyperhomocysteinemia and macromolecule modifications in uremic patients. Clin Chem Lab Med 2005; 43:1032-1038

19 Refsum H, Ueland PM, Nygard O, Vollset SE: Homocysteine and cardiovascular disease. Annu Rev Med 1998;49:31-62.

20 McCully KS, Wilson RB: Homocysteine theory of arteriosclerosis. Atherosclerosis 1975; 22:215-227.

21 McCully KS: Hyperhomocysteinemia and arteriosclerosis: historical perspectives. Clin Chem Lab Med 2005;43:980-986.

22 McCully KS: Vascular pathology of homocysteinemia: implications for the pathogenesis of arteriosclerosis. Am J Pathol 1969;56: 111-128.

-23 Brattström L, Lindgren A, Israelsson B, Malinow MR, Norrving B, Upson B, Hamfelt A: Hyperhomocysteinaemia in stroke: prevalence, cause, and relationships to type of stroke and stroke risk factors. Eur J Clin Invest 1992;22:214-221.
24 Malinow MR, Levenson J, Giral P, Nieto FJ, Razavian M, Segond P, Simon A: Role of blood pressure, uric acid, and hemorheological parameters on plasma homocyst(e)ine concentration. Atherosclerosis 1995; 114: 175-183.

25 Boers GH: Mild hyperhomocysteinemia is an independent risk factor of arterial vascular disease. Semin Thromb Hemost 2000;26: 291-295.

26 Boushey CJ, Beresford SA, Omenn GS, Motulsky AG: A quantitative assessment of plasma homocysteine as a risk factor for vascular disease: probable benefits of increasing folic acid intakes. JAMA 1995;274:10491057.

27 Homocysteine Studies Collaboration: Homocysteine and risk of ischemic heart disease and stroke: a meta-analysis. JAMA 2002;288:2015-2022.

28 Nygard O, NordrehaugJE, Refsum H, Ueland PM, Farstad M, Vollset SE: Plasma homocysteine levels and mortality in patients with coronary artery disease. N Engl J Med 1997; 337:230-236

29 Brattström L, Wilcken DE: Homocysteine and cardiovascular disease: cause or effect? Am J Clin Nutr 2000;72:315-323.

30 Blacher J, Demuth K, Guerin AP, Safar ME, Moatti N, London GM: Influence of biochemical alterations on arterial stiffness in patients with end-stage renal disease. Arterioscler Thromb Vasc Biol 1998; 18:535-541.

31 Bostom AG, Lathrop L: Hyperhomocysteinemia in end-stage renal disease: prevalence, etiology, and potential relationship to arteriosclerotic outcomes. Kidney Int 1997;52: 10-20.

32 Perna AF, Ingrosso D, Castaldo P, De Santo NG, Galletti P, Zappia V: Homocysteine, a new crucial element in the pathogenesis of uremic cardiovascular complications. Miner Electrolyte Metab 1999;25:95-99.

33 Garibotto G, Sofia A, Valli A, Tarroni A, Di Martino M, Cappelli V, Aloisi F, Procopio $\mathrm{V}$ : Causes of hyperhomocysteinemia in patients with chronic kidney diseases. Semin Nephrol 2006;26:3-7. 
-34 Norlund L, Grubb A, Fex G, Leksell H, Nilsson JE, Schenck H, Hultberg B: The increase of plasma homocysteine concentrations with age is partly due to the deterioration of renal function as determined by plasma cystatin C. Clin Chem Lab Med 1998;36:175-178.

-35 Friedman JA, Dwyer JT: Hyperhomocysteinemia as a risk factor for cardiovascular disease in patients undergoing hemodialysis. Nutr Rev 1995;53:197-201.

-36 Moustapha A, Gupta A, Robinson K, Arheart K, Jacobsen DW, Schreiber MJ, Dennis VW: Prevalence and determinants of hyperhomocysteinemia in hemodialysis and peritoneal dialysis. Kidney Int 1999;55:1470-1475.

\37 Garibotto G, Sofia A, Saffioti S, Russo R, Deferrari G, Rossi D, Verzola D, Gandolfo MT, Sala MR: Interorgan exchange of aminothiols in humans. Am J Physiol Endocrinol Metab 2003;284:E757-E763.

\38 Suliman ME, Qureshi AR, Bárány P, Stenvinkel $\mathrm{P}$, Filho JC, Anderstam B, Heimbürger $\mathrm{O}$, Lindholm B, Bergström J: Hyperhomocysteinemia, nutritional status, and cardiovascular disease in hemodialysis patients. Kidney Int 2000;57:1727-1735.

-39 Kalantar-Zadeh K, Block G, Humphreys $\mathrm{MH}$, Kopple JD: Reverse epidemiology of cardiovascular risk factors in maintenance dialysis patients. Kidney Int 2003;63:793808.

-40 Suliman ME, Stenvinkel P, Qureshi AR, Bárány $\mathrm{P}$, Heimbürger $\mathrm{O}$, Anderstam $\mathrm{B}, \mathrm{Al}$ vestrand A, Lindholm B: Hyperhomocysteinemia in relation to plasma free amino acids, biomarkers of inflammation and mortality in patients with chronic kidney disease starting dialysis therapy. Am J Kidney Dis 2004;44:455-465.

-41 Suliman ME, Stenvinkel P, Bárány P, Rashid Qureshi A, Lindholm B: Hyperhomocysteinemia, malnutrition, and inflammation in ESRD patients. Semin Nephrol 2006;26:1419.

-42 Kovesdy CP, Anderson JE, Kalantar-Zadeh $\mathrm{K}$ : Inverse association between lipid levels and mortality in men with chronic kidney disease who are not yet on dialysis: effects of case mix and the malnutrition-inflammation-cachexia syndrome. J Am Soc Nephrol 2007;18:304-311.

43 Kalantar-Zadeh K: Recent advances in understanding the malnutrition-inflammation-cachexia syndrome in chronic kidney disease patients: what is next? Semin Dial 2005; 18:365-369.

44 Deussen A, Pexa A, Loncar R, Stehr SN: Effects of homocysteine on vascular and tissue adenosine: a stake in homocysteine pathogenicity? Clin Chem Lab Med 2005;43:10071010.

45 Chen YF, Li PL, Zou AP: Effect of hyperhomocysteinemia on plasma or tissue adenosine levels and renal function. Circulation 2002;106:1275-1281.
46 Vallon V, Mühlbauer B, Osswald H: Adenosine and kidney function. Physiol Rev 2006; 86:901-940

47 Cramer DV, Wu GD, Chapman FA, Marboe C, Salomon DR: 2-Chlorodeoxyadenosine in combination with cyclosporine inhibits the development of transplant arteriosclerosis in rat cardiac allografts. Transplant Proc 1997; 29:616.

48 Sciotti VM, Van Wylen DG: Attenuation of ischemia-induced extracellular adenosine accumulation by homocysteine. J Cereb Blood Flow Metab 1993;13:208-213.

49 Zou CG, Banerjee R: Homocysteine and redox signaling. Antioxid Redox Signal 2005; 7:547-559.

50 Handy DE, Zhang Y, Loscalzo J: Homocysteine down-regulates cellular glutathione peroxidase (GPx1) by decreasing translation. J Biol Chem 2005;280:15518-15525.

51 Lu SC: Regulation of hepatic glutathione synthesis: current concepts and controversies. FASEB J 1999;13:1169-1183.

52 Mosharov E, Cranford MR, Banerjee R: The quantitatively important relationship between homocysteine metabolism and glutathione synthesis by the transsulfuration pathway and its regulation by redox changes. Biochemistry 2000;39:13005-13011.

53 Zou CG, Banerjee R: Tumor necrosis factoralpha-induced targeted proteolysis of cystathionine beta-synthase modulates redox homeostasis. J Biol Chem 2003;278:1680216808.

54 Cai H, Griendling KK, Harrison DG: The vascular $\mathrm{NAD}(\mathrm{P}) \mathrm{H}$ oxidases as therapeutic targets in cardiovascular diseases. Trends Pharmacol Sci 2003;24:471-478.

55 Geiszt M, Kopp JB, Varnai P, Leto TL: Identification of renox, an NAD(P)H oxidase in kidney. Proc Natl Acad Sci USA 2000;97: 8010-8014.

56 Gill PS, Wilcox CS: NADPH oxidases in the kidney. Antioxid Redox Signal 2006;8:15971607.

57 Griendling KK, Sorescu D, Ushio-Fukai M: NAD $(\mathrm{P}) \mathrm{H}$ oxidase: role in cardiovascular biology and disease. Circ Res 2000;86:494501.

58 Zou AP, Li N, Cowley AW Jr: Production and actions of superoxide in the renal medulla. Hypertension 2001;37(2 Pt 2):547-553.

59 Bedard K, Krause KH: The NOX family of ROS-generating NADPH oxidases: physiology and pathophysiology. Physiol Rev 2007; 87:245-313.

60 Hordijk PL: Regulation of NADPH oxidases: the role of Rac proteins. Circ Res 2006;98: 453-462.

61 Takeya R, Sumimoto H: Regulation of novel superoxide-producing NAD $(\mathrm{P}) \mathrm{H}$ oxidases. Antioxid Redox Signal 2006;8:1523-1532.

62 Shastry S, Ingram AJ, Scholey JW, James LR: Homocysteine induces mesangial cell apoptosis via activation of p38-mitogen-activated protein kinase. Kidney Int 2007;71:304-311.
63 Yi F, Zhang AY, Li N, Muh RW, Fillet M, Renert AF, Li PL: Inhibition of ceramide-redox signaling pathway blocks glomerular injury in hyperhomocysteinemic rats. Kidney Int 2006;70:88-96.

64 Yi P, Melnyk S, Pogribna M, Pogribny IP, Hine RJ, James SJ: Increase in plasma homocysteine associated with parallel increases in plasma S-adenosylhomocysteine and lymphocyte DNA hypomethylation. J Biol Chem 2000;275:29318-29323.

65 Yang ZZ, Zou AP: Homocysteine enhances TIMP-1 expression and cell proliferation associated with NADH oxidase in rat mesangial cells. Kidney Int 2003;63:1012-1020.

66 Yi F, Dos Santos EA, Xia M, Chen QZ, Li PL, Li N: Podocyte injury and glomerulosclerosis in hyperhomocysteinemic rats. Am J Nephrol 2007;27:262-268.

67 Yi F, Zhang AY, Janscha JL, Li PL, Zou AP: Homocysteine activates NADH/NADPH oxidase through ceramide-stimulated Rac GTPase activity in rat mesangial cells. Kidney Int 2004;66:1977-1987.

68 Tyagi N, Sedoris KC, Steed M, Ovechkin AV, Moshal KS, Tyagi SC: Mechanisms of homocysteine-induced oxidative stress. Am J Physiol Heart Circ Physiol 2005;289:H2649H2656.

69 Dai J, Li W, Chang L, Zhang Z, Tang C, Wang N, Zhu Y, Wang X: Role of redox factor-1 in hyperhomocysteinemia-accelerated atherosclerosis. Free Radic Biol Med 2006;41:15661577.

70 Au-Yeung KK, Yip JC, Siow YL, O K: Folic acid inhibits homocysteine-induced superoxide anion production and nuclear factor kappa B activation in macrophages. Can J Physiol Pharmacol 2006;84:141-147.

71 Wolin MS: Interactions of oxidants with vascular signaling systems. Arterioscler Thromb Vasc Biol 2000;20:1430-1442.

72 Kimura S, Zhang GX, Nishiyama A, Shokoji T, Yao L, Fan YY, Rahman M, Abe Y: Mitochondria-derived reactive oxygen species and vascular MAP kinases: comparison of angiotensin II and diazoxide. Hypertension 2005; 45:438-444.

73 Poderoso JJ, Peralta JG, Lisdero CL, Carreras MC, Radisic M, Schöpfer F, Cadenas E, Boveris A: Nitric oxide regulates oxygen uptake and hydrogen peroxide release by the isolated beating rat heart. Am J Physiol 1998; 274(1 Pt 1):C112-C119.

74 Beckman KB, Ames BN: The free radical theory of aging matures. Physiol Rev 1998; 78:547-581.

75 Nemoto S, Takeda K, Yu ZX, Ferrans VJ, Finkel T: Role for mitochondrial oxidants as regulators of cellular metabolism. Mol Cell Biol 2000;20:7311-7318.

76 Sethi AS, Lees DM, Douthwaite JA, Dawnay AB, Corder R: Homocysteine-induced endothelin-1 release is dependent on hyperglycaemia and reactive oxygen species production in bovine aortic endothelial cells. J Vasc Res 2006;43:175-183. 
77 Werstuck GH, Lentz SR, Dayal S, Hossain GS, Sood SK, Shi YY, Zhou J, Maeda N, Krisans SK, Malinow MR, Austin RC: Homocysteine-induced endoplasmic reticulum stress causes dysregulation of the cholesterol and triglyceride biosynthetic pathways. J Clin Invest 2001;107:1263-1273.

78 Roybal CN, Yang S, Sun CW, Hurtado D, Vander Jagt DL, Townes TM, Abcouwer SF: Homocysteine increases the expression of vascular endothelial growth factor by a mechanism involving endoplasmic reticulum stress and transcription factor ATF4. J Biol Chem 2004;279:14844-14852.

-79 Outinen PA, Sood SK, Pfeifer SI, Pamidi S, Podor TJ, Li J, Weitz JI, Austin RC: Homocysteine-induced endoplasmic reticulum stress and growth arrest leads to specific changes in gene expression in human vascular endothelial cells. Blood 1999;94:959967.

-80 Ji C, Kaplowitz N: Hyperhomocysteinemia, endoplasmic reticulum stress, and alcoholic liver injury. World J Gastroenterol 2004;10: 1699-1708.

-81 Kaufman RJ, Scheuner D, Schröder M, Shen X, Lee K, Liu CY, Arnold SM: The unfolded protein response in nutrient sensing and differentiation. Nat Rev Mol Cell Biol 2002;3: 411-421.

82 Kokame K, Agarwala KL, Kato H, Miyata T: Herp, a new ubiquitin-like membrane protein induced by endoplasmic reticulum stress. J Biol Chem 2000;275:32846-32853.

83 Wang XZ, Lawson B, Brewer JW, Zinszner H, Sanjay A, Mi LJ, Boorstein R, Kreibich G, Hendershot LM, Ron D: Signals from the stressed endoplasmic reticulum induce $\mathrm{C} / \mathrm{EBP}$-homologous protein (CHOP/ GADD153). Mol Cell Biol 1996;16:42734280.

84 Cai Y, Zhang C, Nawa T, Aso T, Tanaka M, Oshiro S, Ichijo H, Kitajima S: Homocysteine-responsive ATF3 gene expression in human vascular endothelial cells: activation of c-Jun $\mathrm{NH}_{2}$-terminal kinase and promoter response element. Blood 2000;96:2140-2148.
85 Urano F, Wang X, Bertolotti A, Zhang Y, Chung P, Harding HP, Ron D: Coupling of stress in the ER to activation of JNK protein kinases by transmembrane protein kinase IRE1. Science 2000;287:664-666.

86 Inagi R, Nangaku M, Usuda N, Shimizu A, Onogi H, Izuhara Y, Nakazato K, Ueda Y, Oishi H, Takahashi S, Yamamoto M, Suzuki D, Kurokawa K, van Ypersele de Strihou C, Miyata T: Novel serpinopathy in rat kidney and pancreas induced by overexpression of megsin. J Am Soc Nephrol 2005;16:1339-1349.

87 Miyata T, Nangaku M, Suzuki D, Inagi R, Uragami K, Sakai H, Okubo K, Kurokawa K: A mesangium-predominant gene, megsin, is a new serpin upregulated in IgA nephropathy. J Clin Invest 1998;102:828-836.

88 Inagi R, Nangaku M, Onogi H, Ueyama $\mathrm{H}$, Kitao Y, Nakazato K, Ogawa S, Kurokawa K, Couser WG, Miyata T: Involvement of endoplasmic reticulum (ER) stress in podocyte injury induced by excessive protein accumulation. Kidney Int 2005;68:2639-2650.

89 Jakubowski H: Protein homocysteinylation: possible mechanism underlying pathological consequences of elevated homocysteine levels. FASEB J 1999;13:2277-2283.

90 Perna AF, Satta E, Acanfora F, Lombardi C, Ingrosso D, De Santo NG: Increased plasma protein homocysteinylation in hemodialysis patients. Kidney Int 2006;69:869-876.

91 Jacobsen DW: Homocysteine targeting of plasma proteins in hemodialysis patients. Kidney Int 2006;69:787-789.

92 Jacobsen DW, Catanescu O, Dibello PM, Barbato JC: Molecular targeting by homocysteine: a mechanism for vascular pathogenesis. Clin Chem Lab Med 2005;43:10761083.

93 Perła-Kaján J, Twardowski T, Jakubowski H: Mechanisms of homocysteine toxicity in humans. Amino Acids 2007;32:561-572.

94 Ferretti G, Bacchetti T, Moroni C, Vignini A, Nanetti L, Curatola G: Effect of homocysteinylation of low density lipoproteins on lipid peroxidation of human endothelial cells. Cell Biochem 2004;92:351-360.

$\checkmark 95$ Vignini A, Nanetti L, Bacchetti T, Ferretti G, Curatola G, Mazzanti L: Modification induced by homocysteine and low-density lipoprotein on human aortic endothelial cells: an in vitro study. J Clin Endocrinol Metab 2004;89:4558-4561.
$\$ 96$ Majors AK, Sengupta S, Willard B, Kinter MT, Pyeritz RE, Jacobsen DW: Homocysteine binds to human plasma fibronectin and inhibits its interaction with fibrin. Arterioscler Thromb Vasc Biol 2002;22:13541359.

$\checkmark 97$ Włodek PJ, Smolenski OB, Chwatko G, Iciek MB, Miłkowski A, Bald E, Włodek L: Disruption of thiol homeostasis in plasma of terminal renal failure patients. Clin Chim Acta 2006;366:137-145.

98 Krumdieck CL, Prince CW: Mechanisms of homocysteine toxicity on connective tissues: implications for the morbidity of aging. J Nutr 2000;130(2S suppl):365S-368S.

99 Paizis K, Kirkland G, Khong T, Katerelos M, Fraser S, Kanellis J, Power DA: Heparinbinding epidermal growth factor-like growth factor is expressed in the adhesive lesions of experimental focal glomerular sclerosis. Kidney Int 1999;55:2310-2321.

100 Nakamura T, Ebihara I, Fukui M, Osada S, Nagaoka I, Horikoshi S, Tomino Y, Koide $\mathrm{H}$ : Messenger RNA expression for growth factors in glomeruli from focal glomerular sclerosis. Clin Immunol Immunopathol 1993;66:33-42.

101 Kruman II, Culmsee C, Chan SL, Kruman Y, Guo Z, Penix L, Mattson MP: Homocysteine elicits a DNA damage response in neurons that promotes apoptosis and hypersensitivity to excitotoxicity. J Neurosci 2000;20:6920-6926.

102 Perna AF, Ingrosso D, Zappia V, Galletti P, Capasso G, De Santo NG: Enzymatic methyl esterification of erythrocyte membrane proteins is impaired in chronic renal failure: evidence for high levels of the natural inhibitor S-adenosylhomocysteine. J Clin Invest 1993;91:2497-2503.

103 Perna AF, Ingrosso D, De Santo NG, Galletti P, Zappia V: Mechanism of erythrocyte accumulation of methylation inhibitor Sadenosylhomocysteine in uremia. Kidney Int 1995; 47:247-253.

104 Marcus J, Sarnak MJ, Menon V: Homocysteine lowering and cardiovascular disease risk: lost in translation. Can J Cardiol 2007; 23:707-710. 